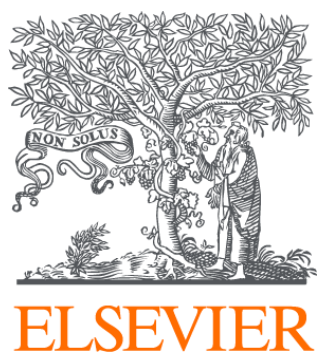

Since January 2020 Elsevier has created a COVID-19 resource centre with free information in English and Mandarin on the novel coronavirus COVID-

19. The COVID-19 resource centre is hosted on Elsevier Connect, the company's public news and information website.

Elsevier hereby grants permission to make all its COVID-19-related research that is available on the COVID-19 resource centre - including this research content - immediately available in PubMed Central and other publicly funded repositories, such as the WHO COVID database with rights for unrestricted research re-use and analyses in any form or by any means with acknowledgement of the original source. These permissions are granted for free by Elsevier for as long as the COVID-19 resource centre remains active. 


\title{
A new Amaryllidaceae alkaloid from the bulbs of Lycoris radiata
}

\author{
HUANG Sheng-Dian ${ }^{1,2}$, ZHANG Yu ${ }^{1}$, HE Hong-Ping ${ }^{1}$, LI Shi-Fei ${ }^{1,2}$, TANG Gui-Hua ${ }^{1,2}$, CHEN \\ Duo-Zhi $^{1,2}$, CAO Ming-Ming ${ }^{1,2}$, DI Ying-Tong ${ }^{1 *}$, HAO Xiao-Jiang ${ }^{1 *}$ \\ ${ }^{1}$ State Key Laboratory of Phytochemistry and Plant Resources in West China, Kunming Institute of Botany, Chinese Academy of \\ Sciences, Kunming 650201, China; \\ ${ }^{2}$ University of the Chinese Academy of Sciences, Beijing 100049, China
}

Available online 20 July 2013

\begin{abstract}
[ABSTRACT] AIM: To study the Amaryllidaceae alkaloids of the bulbs of Lycoris radiata. METHODS: The chemical constituents were isolated and purified by various chromatographic techniques, and the chemical structures were elucidated on the basis of spectroscopic methods. In addition, the antiviral activities of alkaloids 1-10 were evaluated using flu virus A. RESULTS: One new homolycorine-type alkaloid $2 \alpha$-methoxy-6-O-ethyloduline (1), together with nine known alkaloids $2 \alpha$-methoxy-6-O-methyloduline (2), trispherine (3), 8-O-demethylhomolycorine (4), homolycorine (5), 9-O-demethylhomolycorine (6), oduline (7), lycorenine (8), $6 \alpha$ - $O$-methyllycorenine $(\mathbf{9})$ and $O$-ethyllycorenine $(\mathbf{1 0})$ were obtained. CONCLUSION: Alkaloid $\mathbf{1}$ is a new compound, and 1-3 were major alkaloids in this plant. Alkaloids 1-3 showed weak antiviral activities against flu virus A with $\mathrm{IC}_{50}$ values of 2.06, 0.69, and 2.71 $\mu \mathrm{g} \cdot \mathrm{mL}^{-1}$ and $\mathrm{CC}_{50}$ values of $14.37,4.79$, and $80.12 \mu \mathrm{g} \cdot \mathrm{mL}^{-1}$, respectively.
\end{abstract}

[KEY WORDS] Lycoris radiata; Amaryllidaceae alkaloids; Homolycorine-type; $2 \alpha$-Methoxy-6-O-methyloduline; $2 \alpha$-Methoxy-6-O-ethyloduline

[CLC Number] R284.1 [Document code] A [Article ID] 1672-3651(2013)04-0406-005

\section{Introduction}

Amaryllidaceae alkaloids are characteristic constituents of the Amaryllidaceae plant family, whose remarkable biological activities and unique skeletons have attracted great interest as challenging targets for total synthesis and diversity-oriented synthesis ${ }^{[1-9]}$. A series of new Amaryllidaceae alkaloids was isolated from Hosta plantaginea Asch. in a previous phytochemical investigation, which showed some inhibition activities against the tobacco mosaic plant virus (TMV) ${ }^{[10-11]}$. As a continuation of that research work, an

[Received on] 20-Sep.-2012

[Research funding] This project was supported by the National Natural Science Foundation of China (No. 30830114), and Young Academic and Technical Leader Raising Foundation of Yunnan Province to Y.-T. Di (No. 2009CI072)

[*Corresponding author] HAO Xiao-Jiang: Prof., DI Ying-Tong: Prof., Tel: 86-871-65223263, Fax: 86-871-65223070, E-mail: haoxj@mail.kib.ac.cn,diyt@mail.kib.ac.cn

These authors have no conflict of interest to declare. Copyright (c) 2013, China Pharmaceutical University. Published by Elsevier B.V. All rights reserved in-depth phytochemical investigation was conducted on $L y$ coris radiata Herb. (Amaryllidaceae), which is widely distributed in the south of China, Vietnam and Malaysia, and is used as a folk medicine to treat mastitis, tympanitis, ulcers, and carbuncles in China ${ }^{[12]}$. In this work, a new homolycorine-type alkaloid, named $2 \alpha$-methoxy-6-O-ethyloduline (1), together with nine known alkaloids $\mathbf{2 - 1 0}$ (Fig. 1), were isolated from $L$. radiata. Alkaloids 1-3 showed weak antiviral activities against flu virus $\mathrm{A}$ with $\mathrm{IC}_{50}$ values of 2.06, 0.69, and $2.71 \mu \mathrm{g} \cdot \mathrm{mL}^{-1}$ and $\mathrm{CC}_{50}$ values of $14.37,4.79$, and 80.12 $\mu \mathrm{g} \cdot \mathrm{mL}^{-1}$, respectively. Herein, we report the isolation, structure elucidation, and antiviral activities against flu virus A of these ten alkaloids.

\section{Results and Discussion}

$2 \alpha$-Methoxy-6-O-ethyloduline (1) was isolated as a yellow amorphous powder with $[\alpha]_{\mathrm{D}}^{19}+205.7$ (c 2.4, MeOH). The positive HR-ESI-MS displayed an ion peak at $\mathrm{m} / \mathrm{z}$ $360.1811\left([\mathrm{M}+\mathrm{H}]^{+}\right.$, Calcd.: 360.1810$)$, corresponding to the molecular formula $\mathrm{C}_{20} \mathrm{H}_{25} \mathrm{NO}_{5}$, which accounted for nine degrees of unsaturation. The UV spectrum showed absorption bands at 203, 238 and $289 \mathrm{~nm}$, which suggested compound $\mathbf{1}$ 


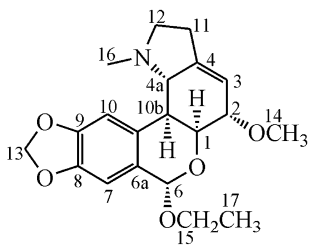

1

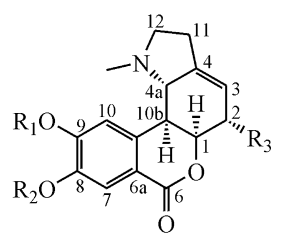

$3 \mathrm{R}_{1}, \mathrm{R}_{2}=-\mathrm{CH}_{2^{-}} ; \mathrm{R}_{3}=\mathrm{OH}$

$4 \mathrm{R}_{1}=\mathrm{Me} ; \mathrm{R}_{2}=\mathrm{H} ; \mathrm{R}_{3}=\mathrm{H}$

$5 \mathrm{R}_{1}, \mathrm{R}_{2}=\mathrm{Me} ; \mathrm{R}_{3}=\mathrm{H}$
$6 \mathrm{R}_{1}=\mathrm{H}, \mathrm{R}_{2}=\mathrm{Me} \quad \mathrm{R}_{3}=\mathrm{H}$

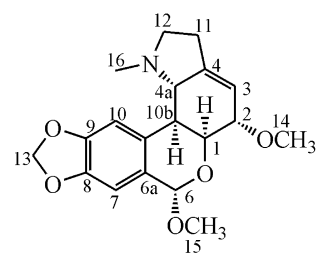

2

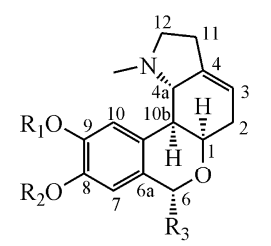

$7 \mathrm{R}_{1}, \mathrm{R}_{2}=-\mathrm{CH}_{2}-; \mathrm{R}_{3}=\mathrm{OH}$

$8 \mathrm{R}_{1}, \mathrm{R}_{2}=\mathrm{Me} ; \mathrm{R}_{3}=\mathrm{OH}$

$9 \mathrm{R}_{1}, \mathrm{R}_{2}=\mathrm{Me} ; \mathrm{R}_{3}=\mathrm{OMe}$

$10 \mathrm{R}_{1}, \mathrm{R}_{2}=\mathrm{Me} ; \mathrm{R}_{3}=\mathrm{OEt}$
Fig. 1 Chemical structures of compounds 1-10

has the same $\mathrm{O}-\mathrm{CH}_{2}$-O-substituted benzene ring as compound 2. Comparison of the NMR data of $\mathbf{1}$ with $\mathbf{2}$ suggested that $\mathbf{1}$ exhibited structural similarities with $\mathbf{2}$. Notable differences in the NMR spectra with those of $\mathbf{2}$ inferred that $\mathbf{1}$ exhibited closely similar signals to $\mathbf{2}$, except for the presence of an OEt group and the disappearance of the OMe group in $\mathbf{2}$. The presence of an OEt group located at C-6 instead of the OMe group in $\mathbf{1}$ was confirmed by the HMBC correlation of $\mathrm{H}-6$ to $\mathrm{C}-15$ and ${ }^{1} \mathrm{H}-{ }^{1} \mathrm{H}$ COSY correlation from $\mathrm{H}-15$ to $\mathrm{H}-17$. Thus, the structure of $\mathbf{1}$ was established and named as $2 \alpha$-methoxy-6-O-ethyloduline.

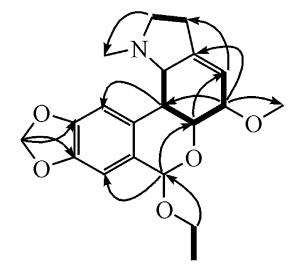

Fig. $2{ }^{1} \mathrm{H}-{ }^{1} \mathrm{H}$ COSY (bold) and selected HMBC (arrow, $\mathrm{H} \rightarrow \mathrm{C})$ correlations of 1

$2 \alpha$-Methoxy-6-O-methyloduline (2) was obtained as a white amorphous powder with $[\alpha]_{\mathrm{D}}^{19}+225.3$ (c 1.6, MeOH). The molecular formula of 2 was determined as $\mathrm{C}_{19} \mathrm{H}_{23} \mathrm{NO}_{5}$ by using HR-ESI-MS ion peak at $m / z 346.1646\left([\mathrm{M}+\mathrm{H}]^{+}\right.$, Calcd: 346.1654 ), with nine degrees of unsaturation. The UV $(\mathrm{MeOH})$ spectrum exhibited the presence of an $\mathrm{O}-\mathrm{CH}_{2}$-O-substituted benzene ring absorption maximum at 203, 238 and $289 \mathrm{~nm}^{[13]}$. The IR (KBr) spectrum displayed absorption bands for a phenyl functional group (1 656, 1608 and $\left.905 \mathrm{~cm}^{-1}\right)$ and a double bond $\left(1562 \mathrm{~cm}^{-1}\right)$. The ${ }^{1} \mathrm{H}$ NMR spectrum (Table) showed one methyl singlet peak at $\delta 2.16$ $(3 \mathrm{H}, \mathrm{s}, \mathrm{H}-16)$, two methoxyl groups at $\delta 3.55(3 \mathrm{H}, \mathrm{s}, \mathrm{H}-15)$ and $\delta 3.43(3 \mathrm{H}, \mathrm{s}, \mathrm{H}-14)$, two aromatic protons at $\delta 6.96(1 \mathrm{H}$, $\mathrm{s}, \mathrm{H}-10)$ and $6.76(1 \mathrm{H}, \mathrm{s}, \mathrm{H}-7)$, a signal for the $\mathrm{H}$-atom of
$\mathrm{C}=\mathrm{C}$ double bond $[\delta 5.46(1 \mathrm{H}, \mathrm{s}, \mathrm{H}-6)]$ and a signal for the $\mathrm{O}-\mathrm{CH}_{2}-\mathrm{O}$ group $[\delta 5.93(2 \mathrm{H}$, br s, H-13)], respectively. The ${ }^{13} \mathrm{C}$ NMR spectrum (Table) revealed 19 signals comprising of five $s p^{2}$ quaternary $\mathrm{C}$-atoms, five $s p^{3} \mathrm{CH}$, three $s p^{2} \mathrm{CH}$ and three $s p^{3} \mathrm{CH}_{2}$ groups, as well as two OMe groups and one NMe group. The five $s p^{2}$ quaternary $\mathrm{C}$-atoms were assignable to four aromatic C-atoms [ $\delta 147.3$ (C-8), 147.1 (C-9), 130.8 (C-6a), $126.9(\mathrm{C}-10 \mathrm{a})]$ and a $\mathrm{C}$-atom of $\mathrm{C}=\mathrm{C}$ double bond $[\delta$ 145.4 (C-4)], 1 of $3 s p^{3} \mathrm{CH}_{2}$ groups was attributable to a O- $\mathrm{CH}_{2}-\mathrm{O}$ group [ $\left.\delta 101.1(\mathrm{C}-13)\right]$, another two $s p^{3} \mathrm{CH}_{2}$ groups corresponded to a $-\mathrm{CH}_{2} \mathrm{CH}_{2}-$ group. The above data proved that $\mathbf{2}$ was a homolycorine-type Amaryllidaceae alkaloid. The NMR data of $\mathbf{2}$ were closely similar to those of the known alkaloid $2 \alpha$-hydroxy-6- $O$-methyloduline ${ }^{[14]}$. The minor difference between them was the presence of an additional methoxyl group signal at $\delta_{\mathrm{C}}(57.2)$ in 2. The HMBC correlation of $\mathrm{H}-2$ to $\mathrm{C}-14$, as well as the downfield shift of C-2 $\left(\delta_{\mathrm{C}} 78.1, \Delta \delta_{\mathrm{C}}+9.8\right)^{[14]}$ confirmed the structure. Thus, the planar structure of $\mathbf{2}$ was elucidated as shown in Fig. 2.

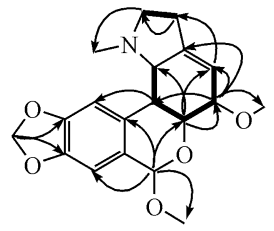

Fig. $3{ }^{1} \mathrm{H}-{ }^{1} \mathrm{H}$ COSY (bold) and selected HMBC (arrow, $\mathrm{H} \rightarrow \mathrm{C}$ ) correlations of 2

The relative configuration of $\mathbf{2}$ was assigned as identical to that of $2 \alpha$-hydroxy-6-O-methyloduline on the basis of the ROESY experiment and coupling constants ${ }^{[14]}$. Detailed analysis of the 2D NMR data established the structure of compound 2 as $2 \alpha$-methoxy-6-O-methyloduline. This is the first time to report the NMR data of this alkaloid ${ }^{[15]}$.

Eight known homolycorine-type alkaloids (3-10) were also isolated and identified as trispherine (3) [14], 8-O-demethylhomolycorine (4) ${ }^{[16]}$, homolycorine (5) ${ }^{[17]}$, 9-O-demethylhomolycorine $(6)^{[18]}$, oduline $(7)^{[19]}$, lycorenine (8) ${ }^{[20]}, 6 \alpha$-O-methyllycorenine (9) ${ }^{[21]}$, $O$-ethyllycorenine (10) ${ }^{[22]}$ by comparison of their $1 \mathrm{D}-\mathrm{NMR}$ data with those in the literature. All of the alkaloids were evaluated for inhibitory activities against flu virus A in vitro ${ }^{[23]}$, and alkaloids 1-3 showed weak antiviral activities with $\mathrm{IC}_{50}$ values of $2.06,0.69$ and $2.71 \mu \mathrm{g} \cdot \mathrm{mL}^{-1}$, and $\mathrm{CC}_{50}$ values of $14.37,4.79$ and $80.12 \mu \mathrm{g} \cdot \mathrm{mL}^{-1}$, respectively.

\section{Experimental}

\subsection{Apparatus and reagents}

Perkin-Elmer model 241 polarmeter; Bio-Rad FTS-135 spectrometer; Shimadzu UV-2401A spectrometer; Bruker AM-400 or a DRX-500 instrument (using TMS as internal standard); Finnigan MAT 90 instrument and VG Auto Spec-3000 spectrometer; silica gel $\left(\mathrm{SiO}_{2}, 300-400\right.$ mesh; 
Qingdao Marine Chemical Co., Ltd., China); MCI gel (CHP20P, 75-150 $\mu \mathrm{m}$; Mitsubishi Chemical Industries Ltd., Japan); Sephadex LH-20 (40-70 $\mu$ m; Amersham Pharmacia Biotech AB, Uppsala, Sweden); Rp-18 gel (150-200 mesh; Merck, Darmstadt, Germany). Semi-Preparative HPLC was performed on a Zorbax SB-C-18 column (i.d. $9.4 \mathrm{~mm} \times 250$ mm; Agilent Co. Ltd., Santa Clara, USA). TLC plates were pre-coated with silica gel GF-254 and HF-254 (Qingdao Marine Chemical Co., Ltd., China).

\subsection{Plant material}

The bulbs of Lycoris radiata were bought from Hengyang, Hunan Province, China, in July 2008, and were identified by GONG Xun. A voucher specimen has been deposited with the Kunming Institute of Botany, Chinese Academy of Sciences, China.

\subsection{Extraction and isolation}

The air-dried and powdered sample (180 kg) was extracted with $\mathrm{MeOH}$ three times to give a crude extract. The crude extract was adjusted to $\mathrm{pH} 2-3$ by dissolving in $0.5 \%$ $\mathrm{HCl}$ soln. The aqueous phase was extracted with EtOAc, and then the acidic $\mathrm{H}_{2} \mathrm{O}$-soluble was adjusted to $\mathrm{pH}$ 9-10 with $10 \%$ aq. $\mathrm{NH}_{3}$ soln. and extracted with $\mathrm{CHCl}_{3}$ to give an alkaline extract $(1.4 \mathrm{~kg})$. The alkaline extract was subjected to $\mathrm{CC}$ $\left(\mathrm{SiO}_{2} ; \mathrm{CHCl}_{3} / \mathrm{MeOH}\right.$ gradient $\left.1: 0 \rightarrow 0: 1\right)$ to afford seven fractions $\left(\mathrm{A}_{1}-\mathrm{A}_{7}\right)$. Fraction $\mathrm{A}_{1}(78 \mathrm{~g})$ was applied to MCI gel $\left(\mathrm{MeOH}-\mathrm{H}_{2} \mathrm{O}, 30 / 70-100 / 0\right)$, Sephadex LH-20 (MeOH), and then to silica gel $\mathrm{CC}$ eluting with petroleum ether(PE)/acetone/diethylamine (5:1:0.05) to yield $3(17 \mathrm{~g}), \mathbf{4}$ (41 mg), 7 (20 mg), and 9 (13 mg), respectively. Fraction $A_{2}$ $(170 \mathrm{~g})$ was subjected to repeated column chromatography (silica gel, Sephadex LH-20 (MeOH), and HPLC) to yield 1 (27 g), 2 (33 g), 10 (11 mg), respectively. Fraction $\mathrm{A}_{3}(15 \mathrm{~g})$ was purified by silica gel and HPLC to give 5 (14 mg), 6 (44 $\mathrm{mg})$ and $\mathbf{8}(8 \mathrm{mg})$, respectively.

$\mathbf{2} \alpha$-Methoxy-6-O-ethyloduline (1) A yellow amorphous powder; $[\alpha]_{\mathrm{D}}^{19}+205.7$ ( c 2.4, MeOH); IR $v_{\max }(\mathrm{KBr}): 3045$, 2 968, 1 738, 1 696, 1 625, 1 563, $925 \mathrm{~cm}^{-1}$; UV (MeOH) $\lambda_{\max }(\log \varepsilon): 289$ (3.59), 238 (3.64), 203 (4.59) nm; ${ }^{1} \mathrm{H}$ NMR and ${ }^{13} \mathrm{C}$ NMR: see Table; HR-ESI-MS $m / z 360.1811$ ([M + $\mathrm{H}]^{+}, \mathrm{C}_{20} \mathrm{H}_{26} \mathrm{NO}_{5}^{+}$; Calcd: 360.1810 ).

2 $\alpha$-Methoxy-6-O-methyloduline (2) A white amorphous powder; $[\alpha]_{\mathrm{D}}^{19}+225.3(c$ 1.6, $\mathrm{MeOH})$; IR $v_{\max }(\mathrm{KBr}): 3$ 060, 2 966, 1 702, 1 678, 1 656, 1 608, $929 \mathrm{~cm}^{-1}$; UV (MeOH) $\lambda_{\max }(\log \varepsilon): 289$ (3.62), 238 (3.65), 203 (4.61) nm; ${ }^{1} \mathrm{H}$ NMR and ${ }^{13} \mathrm{C}$ NMR: see Table; HR-ESI-MS: $346.1646\left([\mathrm{M}+\mathrm{H}]^{+}\right.$, $\mathrm{C}_{19} \mathrm{H}_{24} \mathrm{NO}_{5}^{+}$; Calcd.: 346.1654 ).

Trispherine (3) A white amorphous powder; ESI-MS $m / z 315.1[\mathrm{M}+\mathrm{H}]^{+}, \mathrm{C}_{17} \mathrm{H}_{17} \mathrm{NO}_{5} ;{ }^{1} \mathrm{H}$ NMR (400 MHz, $\mathrm{CDCl}_{3}$ ) $\delta: 4.39(1 \mathrm{H}, \mathrm{s}, \mathrm{H}-1), 3.47$ (1H, s, H-2), $5.65(1 \mathrm{H}, \mathrm{s}, \mathrm{H}-3), 2.90$ $(1 \mathrm{H}, \mathrm{d}, J=8.3 \mathrm{~Hz}, \mathrm{H}-4 \mathrm{a}), 6.74(1 \mathrm{H}, \mathrm{s}, \mathrm{H}-7), 6.95(1 \mathrm{H}, \mathrm{s}$, $\mathrm{H}-10), 2.63(1 \mathrm{H}, \mathrm{d}, J=9.0, \mathrm{H}-10 \mathrm{~b}), 2.53(2 \mathrm{H}, \mathrm{d}, J=8.0$, $\mathrm{H}-11), 3.15$ (2H, t, $J=7.6, \mathrm{H}-12), 6.05$ (2H, br. s, $-\mathrm{OCH}_{2} \mathrm{O}-$ ),
$4.60(1 \mathrm{H}, \mathrm{s},-\mathrm{OH}), 2.04\left(3 \mathrm{H}, \mathrm{s},-\mathrm{NCH}_{3}\right) ;{ }^{13} \mathrm{C} \mathrm{NMR}(100 \mathrm{MHz}$, $\left.\mathrm{CDCl}_{3}\right) \delta: 67.1(\mathrm{C}-1), 82.2(\mathrm{C}-2), 118.2$ (C-3), 139.3 (C-4), 66.8 (C-4a), 164.7 (C-6), 118.4 (C-6a), 108.7 (C-7), 147.9 (C-8), 151.8 (C-9), 109.8 (C-10), 139.3 (C-10a), 39.7 (C-10b), 27.7 (C-11), 56.0 (C-12), 102.1 (-OCH $\left.\mathrm{OC}_{2} \mathrm{O}\right)$, $43.5\left(-\mathrm{NCH}_{3}\right)$.

8-O-Demethylhomolycorine (4) Yellow oil; ESI-MS $m / z 301.1[\mathrm{M}+\mathrm{H}]{ }^{+}, \mathrm{C}_{17} \mathrm{H}_{19} \mathrm{NO}_{4} ;{ }^{1} \mathrm{H}$ NMR $\left(400 \mathrm{MHz}, \mathrm{CDCl}_{3}\right)$ $\delta: 4.77(1 \mathrm{H}, \mathrm{s}, \mathrm{H}-1), 3.47(1 \mathrm{H}, \mathrm{s}, \mathrm{H}-2), 5.51(1 \mathrm{H}, \mathrm{s}, \mathrm{H}-3), 3.15$ $(1 \mathrm{H}, \mathrm{dd}, J=13.0,5.4 \mathrm{~Hz}, \mathrm{H}-4 \mathrm{a}), 7.01(1 \mathrm{H}, \mathrm{s}, \mathrm{H}-7), 7.55(1 \mathrm{H}$, s, H-10), $2.62(1 \mathrm{H}, \mathrm{d}, J=8.3 \mathrm{~Hz}, \mathrm{H}-10 \mathrm{~b}), 2.51(2 \mathrm{H}, \mathrm{d}, J=9.8$ $\mathrm{Hz}, \mathrm{H}-11), 3.13$ (2H, dd, $J=8.5,3.5 \mathrm{~Hz}, \mathrm{H}-12), 3.92$ (3H, br. $\left.\mathrm{s},-\mathrm{OCH}_{3}\right), 3.82(1 \mathrm{H}, \mathrm{s},-\mathrm{OH}), 2.01\left(3 \mathrm{H}, \mathrm{s},-\mathrm{NCH}_{3}\right) ;{ }^{13} \mathrm{C} \mathrm{NMR}$ $\left(100 \mathrm{MHz}, \mathrm{CDCl}_{3}\right) \delta: 67.6(\mathrm{C}-1), 31.2(\mathrm{C}-2), 116.1(\mathrm{C}-3)$, 136.4 (C-4), 66.6 (C-4a), 165.7 (C-6), 117.4 (C-6a), 110.4 (C-7), 145.8 (C-8), 151.2 (C-9), 115.6 (C-10), 140.3 (C-10a), 43.6 (C-10b), 27.9 (C-11), 56.4 (C-12), $56.2\left(-\mathrm{OCH}_{3}\right), 43.6$ $\left(-\mathrm{NCH}_{3}\right)$.

Homolycorine (5) A yellow amorphous powder; ESI-MS $m / z 315.2[\mathrm{M}+\mathrm{H}]^{+}, \mathrm{C}_{18} \mathrm{H}_{21} \mathrm{NO}_{4} ;{ }^{1} \mathrm{H}$ NMR (400 $\left.\mathrm{MHz}, \mathrm{CDCl}_{3}\right) \delta: 4.81(1 \mathrm{H}, \mathrm{ddd}, J=4.8,1.8,1.7 \mathrm{~Hz}, \mathrm{H}-1)$, 2.49 (1H, m, H-2), 5.50 (1H, m, H-3), $2.72(1 \mathrm{H}, \mathrm{dd}, J=9.6$, $2.0 \mathrm{~Hz}, \mathrm{H}-4 \mathrm{a}), 7.57$ (1H, s, H-7), 6.99 (1H, s, H-10), $2.64(1 \mathrm{H}$, dd, $J=9.6,1.8 \mathrm{~Hz}, \mathrm{H}-10 \mathrm{~b}), 2.63(2 \mathrm{H}, \mathrm{m}, \mathrm{H}-11), 3.14(1 \mathrm{H}$, ddd, $J=10.0,7.0,3.5 \mathrm{~Hz}, \mathrm{H} \alpha-12), 2.24(1 \mathrm{H}, \mathrm{dd}, J=18.0,9.2$ $\mathrm{Hz}, \mathrm{H} \beta-12), 3.92\left(3 \mathrm{H}, \mathrm{s},-\mathrm{OCH}_{3}\right), 3.95\left(3 \mathrm{H}, \mathrm{s},-\mathrm{OCH}_{3}\right), 2.00$ $\left(3 \mathrm{H}, \mathrm{s},-\mathrm{NCH}_{3}\right) ;{ }^{13} \mathrm{C} \mathrm{NMR}\left(100 \mathrm{MHz}, \mathrm{CDCl}_{3}\right) \delta: 66.8(\mathrm{C}-1)$, 31.1 (C-2), 115.9 (C-3), 137.3 (C-4), 66.4 (C-4a), 165.8 (C-6), 116.7 (C-6a), 110.8 (C-7), 148.9 (C-8), 153.1 (C-9), 115.9 (C-10), 137.3 (C-10a), 43.6 (C-10b), 27.9 (C-11), 56.4 (C-12), $56.1\left(-\mathrm{OCH}_{3}\right), 56.4\left(-\mathrm{OCH}_{3}\right), 43.6\left(-\mathrm{NCH}_{3}\right)$.

9-O-Demethylhomolycorine (6) A white amorphous powder; ESI-MS $m / z$ 301.1 $[\mathrm{M}+\mathrm{H}]^{+}, \mathrm{C}_{17} \mathrm{H}_{19} \mathrm{NO}_{4} ;{ }^{1} \mathrm{H}$ NMR $\left(400 \mathrm{MHz}, \mathrm{CDCl}_{3}\right) \delta: 4.80(1 \mathrm{H}, \mathrm{ddd}, J=4.8,1.7,1.6 \mathrm{~Hz}$, $\mathrm{H}-1), 2.51(1 \mathrm{H}, \mathrm{m}, \mathrm{H}-2), 5.55(1 \mathrm{H}, \mathrm{m}, \mathrm{H}-3), 2.71(1 \mathrm{H}, \mathrm{d}, J=$ $10.0 \mathrm{~Hz}, \mathrm{H}-4 \mathrm{a}), 7.54$ (1H, s, H-7), 6.91 (1H, s, H-10), 2.60 (1H, dd, $J=10.0,1.6 \mathrm{~Hz}, \mathrm{H}-10 \mathrm{~b}), 2.61$ (2H, m, H-11), 3.15 (1H, ddd, $J=10.0,7.0,3.5 \mathrm{~Hz}, \mathrm{H} \alpha-12), 2.30(1 \mathrm{H}, \mathrm{dd}, J=$ $18.3,9.2 \mathrm{~Hz}, \mathrm{H} \beta-12), 3.94\left(3 \mathrm{H}, \mathrm{s},-\mathrm{OCH}_{3}\right), 2.01(3 \mathrm{H}, \mathrm{s}$, $\left.-\mathrm{NCH}_{3}\right) ;{ }^{13} \mathrm{C} \mathrm{NMR}\left(100 \mathrm{MHz}, \mathrm{CDCl}_{3}\right) \delta: 77.5(\mathrm{C}-1), 31.2$ (C-2), 116.0 (C-3), 140.4 (C-4), 77.3 (C-4a), 165.6 (C-6), 117.5 (C-6a), 110.3 (C-7), 145.7 (C-8), 151.0 (C-9), 115.5 (C-10), 136.5 (C-10a), 43.6 (C-10b), 27.9 (C-11), 56.4 (C-12), $56.3\left(-\mathrm{OCH}_{3}\right), 43.8\left(-\mathrm{NCH}_{3}\right)$.

Oduline (7) A white amorphous powder; ESI-MS $\mathrm{m} / \mathrm{z}$ $301.1[\mathrm{M}+\mathrm{H}]^{+}, \mathrm{C}_{17} \mathrm{H}_{19} \mathrm{NO}_{4} ;{ }^{1} \mathrm{H}$ NMR (400 MHz, MeOD) $\delta$ : $4.35(1 \mathrm{H}, \mathrm{d}, J=6.0 \mathrm{~Hz}, \mathrm{H}-1), 2.31(1 \mathrm{H}, \mathrm{dm}, J=19.0,2.8 \mathrm{~Hz}$, $\mathrm{H} \alpha-2), 2.62(1 \mathrm{H}, \mathrm{d}, J=19.3 \mathrm{~Hz}, \mathrm{H} \beta-2), 5.46(1 \mathrm{H}, \mathrm{br} \mathrm{d}, J=$ $2.9 \mathrm{~Hz}, \mathrm{H}-3), 2.71(1 \mathrm{H}$, br. d, $J=9.5 \mathrm{~Hz}, \mathrm{H}-4 \mathrm{a}), 5.99(1 \mathrm{H}, \mathrm{s}$, H-6), $6.85(1 \mathrm{H}, \mathrm{s}, \mathrm{H}-7), 6.90(1 \mathrm{H}, \mathrm{s}, \mathrm{H}-10), 2.46(1 \mathrm{H}, \mathrm{m}$, $\mathrm{H}-10 \mathrm{~b}), 2.64$ (2H, m, H-11), 3.14 (1H, ddd, $J=9.2,6.3,3.8 \mathrm{~Hz}$, $\mathrm{H} \alpha-12), 2.25$ (1H, dd, $J=18.7,9.5 \mathrm{~Hz}, \mathrm{H} \beta-12), 5.97(2 \mathrm{H}, \mathrm{d}, \mathrm{J}$ $\left.=14.7 \mathrm{~Hz},-\mathrm{OCH}_{2} \mathrm{O}-\right), 3.67(1 \mathrm{H}, \mathrm{s},-\mathrm{OH}), 2.11\left(3 \mathrm{H}, \mathrm{s},-\mathrm{NCH}_{3}\right)$; ${ }^{13} \mathrm{C}$ NMR (100 MHz, MeOD) $\delta: 66.7$ (C-1), 31.7 (C-2), 115.7 
Table ${ }^{1} \mathrm{H}$ and ${ }^{13} \mathrm{C}$ NMR Data (500 and $100 \mathrm{MHz}$ ) of alkaloids 1 and $2(\mathrm{~J}$ in $\mathrm{Hz})$ in $\mathrm{CDCl}_{3}$

\begin{tabular}{|c|c|c|c|c|}
\hline \multirow{2}{*}{ Position } & \multicolumn{2}{|c|}{1} & \multicolumn{2}{|c|}{2} \\
\hline & $\delta_{\mathrm{H}}$ & $\delta_{\mathrm{C}}$ & $\delta_{\mathrm{H}}$ & $\delta_{\mathrm{C}}$ \\
\hline 1 & $4.28, \mathrm{~s}$ & 69.2 & $4.23, \mathrm{~s}$ & 69.2 \\
\hline 2 & $3.70, \mathrm{~s}$ & 78.3 & $3.76, \mathrm{~s}$ & 78.1 \\
\hline 3 & $5.61, \mathrm{~s}$ & 116.6 & $5.61, \mathrm{~s}$ & 117.4 \\
\hline 4 & & 146.5 & & 145.1 \\
\hline $4 a$ & $2.70, \mathrm{~d}(8.7)$ & 67.6 & $2.71, \mathrm{~d}(8.3)$ & 67.8 \\
\hline 6 & 5.58, br s & 97.4 & $5.46, \mathrm{~s}$ & 98.6 \\
\hline $6 a$ & & 127.1 & & 126.9 \\
\hline 7 & 6.76, br s & 107.3 & $6.75, \mathrm{~s}$ & 107.3 \\
\hline 8 & & 147.1 & & 147.3 \\
\hline 9 & & 147.0 & & 147.1 \\
\hline 10 & $6.88, \mathrm{br} \mathrm{s}$ & 110.0 & $6.89, \mathrm{~s}$ & 110.1 \\
\hline $10 \mathrm{a}$ & & 131.2 & & 130.8 \\
\hline $10 \mathrm{~b}$ & $2.47, \mathrm{~d}(2.2)$ & 41.3 & $2.71, \mathrm{~d}(10.8)$ & 40.6 \\
\hline 11 & $2.49, \mathrm{~d}(7.9)$ & 28.3 & $2.50, \mathrm{~d}(7.9)$ & 28.1 \\
\hline 12 & $\begin{array}{l}3.18, \mathrm{dd}(9.7,6.1) \\
2.27 \text {, dd }(9.7,6.1)\end{array}$ & 56.7 & $3.17, \mathrm{~s}$ & 56.6 \\
\hline 13 & 5.96, br s & 101.0 & 5.95, br s & 101.1 \\
\hline 14 & 3.43 , br s & 57.1 & $3.43, \mathrm{~s}$ & 57.1 \\
\hline 15 & $\begin{array}{l}3.92, \mathrm{~m} \\
3.94, \mathrm{~m}\end{array}$ & 63.6 & $3.55, \mathrm{~s}$ & 55.4 \\
\hline 16 & $2.12, \mathrm{br} \mathrm{s}$ & 44.3 & $2.11, \mathrm{~s}$ & 44.0 \\
\hline 17 & $1.30, \operatorname{td}(7.0,3.6)$ & 15.4 & & \\
\hline
\end{tabular}

(C-3), 140.6 (C-4), 67.5 (C-4a), 91.8 (C-6), 132.0 (C-6a), 107.4 (C-7), 147.0 (C-8), 147.0 (C-9), 109.8 (C-10), 128.2 (C-10a), 44.0 (C-10b), 28.1 (C-11), 56.7 (C-12), 101.0 $\left(-\mathrm{OCH}_{2} \mathrm{O}-\right), 44.3\left(-\mathrm{NCH}_{3}\right)$.

Lycorenine (8) A white amorphous powder; ESI-MS $\mathrm{m} / \mathrm{z}$ $317.2[\mathrm{M}+\mathrm{H}]^{+}, \mathrm{C}_{18} \mathrm{H}_{23} \mathrm{NO}_{4} ;{ }^{1} \mathrm{H}$ NMR (400 MHz, $\mathrm{CDCl}_{3}$ : MeOD $1: 1) \delta: 4.53(1 \mathrm{H}, \mathrm{d}, J=5.4 \mathrm{~Hz}, \mathrm{H}-1), 2.50(1 \mathrm{H}, \mathrm{m}$, $\mathrm{H}-2), 5.70(1 \mathrm{H}, \mathrm{s}, \mathrm{H}-3), 2.68$ (1H, br. d, $J=9.7 \mathrm{~Hz}, \mathrm{H}-4 \mathrm{a})$, $6.12(1 \mathrm{H}, \mathrm{s}, \mathrm{H}-6), 7.08$ (1H, s, H-7), 7.15 (1H, s, H-10), 2.53 (1H, m, H-10b), 2.67 (2H, m, H-11), $3.05(1 \mathrm{H}, \mathrm{d}, J=8.7 \mathrm{~Hz}$, $\mathrm{H} \alpha-12), 2.31(1 \mathrm{H}, \mathrm{m}, \mathrm{H} \beta-12), 4.04\left(3 \mathrm{H}, \mathrm{s},-\mathrm{OCH}_{3}\right), 4.05(3 \mathrm{H}$, $\left.\mathrm{s},-\mathrm{OCH}_{3}\right), 3.50(1 \mathrm{H}, \mathrm{s},-\mathrm{OH}), 2.13\left(3 \mathrm{H}, \mathrm{s},-\mathrm{NCH}_{3}\right) ;{ }^{13} \mathrm{C} \mathrm{NMR}$ (100 MHz, $\left.\mathrm{CDCl}_{3}: \mathrm{MeOD} 1: 1\right) \delta: 67.1$ (C-1), 32.1 (C-2), 117.6 (C-3), 139.4 (C-4), 68.4 (C-4a), 91.9 (C-6), 130.4 (C-6a), 111.0 (C-7), 149.0 (C-8), 149.0 (C-9), 113.0 (C-10), 128.1 (C-10a), 43.6 (C-10b), 28.2 (C-11), 57.1 (C-12), 56.2 $\left(-\mathrm{OCH}_{3}\right), 56.3\left(-\mathrm{OCH}_{3}\right), 44.0\left(-\mathrm{NCH}_{3}\right)$.

6a-O-Methyllycorenine (9) A white amorphous powder; ESI-MS $m / z 331.2[\mathrm{M}+\mathrm{H}]^{+}, \mathrm{C}_{19} \mathrm{H}_{25} \mathrm{NO}_{4} ;{ }^{1} \mathrm{H}$ NMR (400 $\mathrm{MHz}, \mathrm{CDCl}_{3}$ : MeOD $\left.1: 1\right) \delta: 4.13(1 \mathrm{H}, \mathrm{d}, J=4.2 \mathrm{~Hz}, \mathrm{H}-1)$, $2.52(1 \mathrm{H}, \mathrm{m}, \mathrm{H}-2), 5.71(1 \mathrm{H}, \mathrm{s}, \mathrm{H}-3), 2.65(1 \mathrm{H}, \mathrm{t}, J=2.40$ Hz, H-4a), 5.71 (1H, s, H-6), 6.87 (1H, s, H-7), 6.98 (1H, s, $\mathrm{H}-10), 2.54$ (1H, m, H-10b), 2.64 (2H, m, H-11), $3.27(2 \mathrm{H}$, $\mathrm{d}, J=8.0 \mathrm{~Hz}, \mathrm{H}-12), 3.82\left(3 \mathrm{H}, \mathrm{s},-\mathrm{OCH}_{3}\right), 3.85(3 \mathrm{H}, \mathrm{s}$, $\left.-\mathrm{OCH}_{3}\right), 4.86\left(3 \mathrm{H}, \mathrm{s},-\mathrm{OCH}_{3}\right), 2.20\left(3 \mathrm{H}, \mathrm{s},-\mathrm{NCH}_{3}\right) ;{ }^{13} \mathrm{C}$ NMR (100 MHz, $\left.\mathrm{CDCl}_{3}: \mathrm{MeOD} 1: 1\right) \delta: 67.1(\mathrm{C}-1), 31.9$ (C-2), 117.9 (C-3), 138.6 (C-4), 68.7 (C-4a), 98.9 (C-6),
130.2 (C-6a), 111.0 (C-7), 149.1 (C-8), 149.1 (C-9), 113.1 (C-10), 126.3 (C-10a), 42.8 (C-10b), 28.1 (C-11), 56.9 $(\mathrm{C}-12), 55.7\left(-\mathrm{OCH}_{3}\right), 56.3\left(-\mathrm{OCH}_{3}\right), 55.4\left(-\mathrm{OCH}_{3}\right), 43.9$ $\left(-\mathrm{NCH}_{3}\right)$.

$\boldsymbol{O}$-Ethyllycorenine (10) A white amorphous powder; ESI-MS $m / z 345.2[\mathrm{M}+\mathrm{H}]^{+}, \mathrm{C}_{20} \mathrm{H}_{27} \mathrm{NO}_{4} ;{ }^{1} \mathrm{H}$ NMR (400 $\left.\mathrm{MHz}, \mathrm{CDCl}_{3}\right) \delta: 4.24(1 \mathrm{H}, \mathrm{d}, J=4.80 \mathrm{~Hz}, \mathrm{H}-1), 2.44(1 \mathrm{H}, \mathrm{d}$, $J=1.40, \mathrm{H}-2), 5.50(1 \mathrm{H}, \mathrm{s}, \mathrm{H}-3), 2.43(1 \mathrm{H}, \mathrm{d}, J=1.42 \mathrm{~Hz}$, H-4a), 5.51 (1H, s, H-6), $6.86(1 \mathrm{H}, \mathrm{s}, \mathrm{H}-7), 6.97(1 \mathrm{H}, \mathrm{s}$, $\mathrm{H}-10), 2.43$ (1H, s, H-10b), 2.45 (2H, m, H-11), 3.17 (1H, s, $\mathrm{H}-12), 3.82\left(3 \mathrm{H}, \mathrm{s},-\mathrm{OCH}_{3}\right), 3.84\left(3 \mathrm{H}, \mathrm{s},-\mathrm{OCH}_{3}\right), 2.43(2 \mathrm{H}, \mathrm{q}$, $\left.J=9.05,1.45 \mathrm{~Hz},-\mathrm{OCH}_{2} \mathrm{CH}_{3}\right), 2.31(3 \mathrm{H}, \mathrm{t}, J=2.50 \mathrm{~Hz}$, $\left.-\mathrm{OCH}_{2} \mathrm{CH}_{3}\right), 2 . .08\left(3 \mathrm{H}, \mathrm{s},-\mathrm{NCH}_{3}\right) ;{ }^{13} \mathrm{C} \mathrm{NMR}(100 \mathrm{MHz}$, $\mathrm{CDCl}_{3}$ ) $\delta: 66.7$ (C-1), 31.7 (C-2), 116.1 (C-3), 138.6 (C-4), 67.7 (C-4a), 97.2 (C-6), 130.2 (C-6a), 111.1 (C-7), 148.4 (C-8), 148.4 (C-9), 112.7 (C-10), 126.0 (C-10a), 43.8 (C-10b), $28.1 \quad(\mathrm{C}-11), \quad 56.8 \quad(\mathrm{C}-12), \quad 63.5 \quad\left(-\mathrm{OCH}_{2} \mathrm{CH}_{3}\right), \quad 15.5$ $\left(-\mathrm{OCH}_{2} \mathrm{CH}_{3}\right), 55.8\left(-\mathrm{OCH}_{3}\right), 56.1\left(-\mathrm{OCH}_{3}\right), 44.2\left(-\mathrm{NCH}_{3}\right)$.

\section{Acknowledgements}

We thank Prof. GONG Xun for the identification of the plant material.

\section{References}

[1] Martin SF, Garrison PJ. General methods for alkaloid synthesis. Total synthesis of racemic lycoramine [J]. J Org Chem, 1982, 
47(10): 1513-1518

[2] Schultz AG, Yee YK, Berger MH. Total synthesis of $d l$-lycoramine [J]. J Am Chem Soc, 1977, 99(24): 8065-8067.

[3] Misaka Y, Mizutani T, Sekido M, et al. The total synthesis of (土)-lycoramine. Part II [J]. J Chem Soc, Sec C, 1968, 23: 2954-2959.

[4] Hazama N, Irie H, Mizutani $\mathrm{T}$, et al. Total synthesis of (+)-lycoramine. I [J]. J Chem Soc, Sec C, 1968, 23: 2947-2953.

[5] Barton DHR, Kirby GW. Phenol oxidation and biosynthesis.V. Synthesis of galanthamine [J]. J Chem Soc, 1962: 806-817.

[6] Parker KA, Kim HJ. A short stereospecific synthesis of $(d l)$-lycoramine. Control of relative stereochemistry by dipole effects [J]. J Org Chem, 1992, 57(2): 752-755.

[7] Kita Y, Arisawa M, Gyoten M, et al. Oxidative intramolecular phenolic coupling reaction induced by a hypervalent iodine (III) reagent: Leading to galanthamine-type Amaryllidaceae alkaloids [J]. J Org Chem, 1998, 63(19): 6625-6633.

[8] Sanchez IH, Soria JJ, Lopez FJ, et al. Total synthesis of racemic lycoramine [J]. J Org Chem, 1984, 49(1): 157-163.

[9] Li SY, Chen C, Zhang HQ, et al. Identification of natural compounds with antiviral activities against SARS-associated coronavirus [J]. Antiviral Res, 2005, 67(1): 18-27.

[10] Wang YH, Zhang ZK, Yang FM, et al. Benzylphenethylamine Alkaloids from Hosta plantaginea with inhibitory activity against tobacco mosaic virus and acetylcholinesterase $[\mathrm{J}]$. J Nat Prod, 2007, 70(9): 1458-1461.

[11] Wang YH, Gao S, Yang FM, et al. Structure elucidation and biomimetic synthesis of hostasinine A, a new benzylphenethylamine alkaloid from Hosta plantaginea [J]. Org Lett, 2007, 9(25): 5279-5281.

[12] Wang FZ, Tang J, In: Flora Reipublicae Popularis Sinicae [M]. Wu ZY, Eds; Beijing: Science Press, 1980, 14: 49-52.
[13] Vazquez Tato MP, Castedo L, Riguera R. New alkaloids from Pancratium maritimum L. [J]. Heterocycles, 1988, 27(12): 2833-2838.

[14] Almanza GR, Fernandez JM, Wakori EWT, et al. Alkaloids from Narcissus cv. Salome [J]. Phytochemistry, 1996, 43(6): 1375-1378.

[15] Liao N, Ao MZ, Zhang P, et al. Extracts of Lycoris aurea induce apoptosis in murine sarcoma S180 cells [J]. Molecules, 2012, 17(4): 3723-3735.

[16] Strahil B, Ricardo RC, Carles C, et al. Revised NMR data for incartine: an alkaloid from Galanthus elwesii [J]. Molecules, 2007, 12(7): 1430-1435.

[17] Suau R, Gomez AI, Rico R, et al. Alkaloid $N$-oxides of Amaryllidaceae [J]. Phytochemistry, 1988, 27(10): 3285-3287.

[18] Bastida J, Llabres JM, Viladomat F, et al. Narcissus alkaloids. Part III. 9-O-demethylhomolycorine from Narcissus confusus [J]. J Nat Prod, 1987, 50(2): 199-202.

[19] Kreh M, Matusch R. $O$-Methyloduline and $N$-demethylmasonine, alkaloids from Narcissus pseudonarcissus [J]. Phytochemistry, 1995, 38(6): 1533-1535.

[20] Codina C, Viladomat F, Bastida J, et al. 2D NMR studies of lycorenine as a model for the structural assignment of lycorenine-type alkaloids [J]. Nat Prod Lett, 1992, 1(2): 85-92.

[21] Codina C, Bastida J, Viladomat F, et al. Alkaloids from Narcissus munozii-garmendiae [J]. Phytochemistry, 1993, 32(5): 1354-1356.

[22] Kreh M, Matusch R, Witte L. Capillary gas chromatography-mass spectrometry of Amaryllidaceae alkaloids [J]. Phytochemistry, 1995, 38(3): 773-776.

[23] Liu C, Liu LF, Wang B, et al. A review on 2009 influenza A virus [J]. Agric Sci Tech, 2012, 13(2): 424-427.

\section{红花石蒜中的一个新石蒜生物碱}

黄升殿 ${ }^{1,2}$ ，张 于 ${ }^{1}$ ，何红平 ${ }^{1}$ ，李石飞 ${ }^{1,2}$ ，唐贵华 ${ }^{1,2}$ ，陈铎之 ${ }^{1,2}$ ，曹明明 1,2 ，邸迎䑣 $1^{*}$, 郝小江 $1 *$

1 中国科学院昆明植物研究所植物化学与西部植物资源持续利用国家重点实验室, 昆明 650201;

2 中国科学院大学, 北京 100049

【摘要】目的: 研究红花石蒜中的石蒜生物碱。方法: 采用多种层析柱分离手段, 运用 NMR 和 HR-ESI-MS 等波谱技术 鉴定化合物的结构。此外, 生物碱 1-10 进行了流感甲型病毒的活性测试。结果: 从红花石蒜中分离鉴定了 1 个新石蒜生物碱和 9 个已知的石蒜生物碱: $2 \alpha$-methoxy-6-O-ethyloduline (1), $2 \alpha$-methoxy-6- $O$-methyloduline (2), trispherine (3), 8- $O$-demethylhomolycorine (4), homolycorine (5), 9-O-demethylhomolycorine (6), oduline (7), lycorenine (8), 6 $\alpha$ - $O$-methyllycorenine (9) 和 $O$-ethyllycorenine (10)。结论: 化合物 1 为新的高石蒜碱类型的石蒜生物碱, 生物碱 1-3 为该植物中的主要成分, 且对流感甲型 病毒显示了较弱的抗病毒活性, $\mathrm{IC}_{50}$ 分别为 $2.06,0.69,2.71 \mu \mathrm{g} \cdot \mathrm{mL}^{-1}, \mathrm{CC}_{50}$ 分别为 $14.37,4.79,80.12 \mu \mathrm{g} \cdot \mathrm{mL}^{-1}$ 。

【关键词】红花石蒜; 石蒜生物碱; 高石蒜碱类型; $2 \alpha$-methoxy-6-O-methyloduline; $2 \alpha$-methoxy-6-O-ethyloduline

【基金项目】国家自然科学基金重点项目(No. 30830114), 云南省学术与技术带头人后备人才项目(No. 2009CI072)资助 